FEDSM2021-65225

\title{
DRAFT: FREQUENCY RESPONSE OF SYNTHETIC JETS EMANATING FROM AN ARRAY OF CIRCULAR ORIFICES
}

\author{
Nadim Arafa* \\ Postdoctoral Fellow \\ Institute for Aerospace Studies \\ University of Toronto \\ nadim.arafa@utoronto.ca
}

\author{
Pierre Sullivan \\ Professor \\ Mechanical and Industrial \\ Engineering \\ University of Toronto \\ sullivan@mie.utoronto.ca
}

\author{
Alis Ekmekci \\ Associate Professor \\ Institute for Aerospace Studies \\ University of Toronto \\ Ekmekci@utias.utoronto.ca
}

\begin{abstract}
The effect of the excitation frequency of synthetic jet actuators on the mean jet velocity of synthetic jets issuing from an array of circular orifices is investigated experimentally. Herein, the focus is placed on an array of circular orifices, rather than a single orifice, as it brings the advantage of covering long-span airfoils. The array consists of 16 circular orifices, each having a diameter of $3.42 \mathrm{~mm}$, distributed over a span of $300 \mathrm{~mm}$. The jets are generated by the excitation of a single cavity via 16 piezoelectric elements. Localized velocity measurements at the exit of the orifices show that the mean jet velocity varies with the excitation frequency. Several distinct resonant peaks were observed in the frequency response. Acoustic simulations of the cavity showed that these peaks correspond to acoustic mode shapes of the cavity. Due to the high-aspect ratio of the cavity, several acoustic mode shapes exist in the excitation frequency range aside from the Helmholtz resonance frequency. Moreover, the mean jet velocity emanating from the array shows a variation from orifice to orifice, depending on the excited acoustic mode.
\end{abstract}

\section{NOMENCLATURE}

$D$ Orifice diameter [m]

$E$ Input voltage peak-to-peak $\left[\mathrm{V}_{\mathrm{pp}}\right]$

\footnotetext{
*Address all correspondence to this author.
}

$f_{e} \quad$ Excitation frequency $[\mathrm{Hz}]$

$f_{s} \quad$ Resonance frequency $[\mathrm{Hz}]$

$n$ Integer showing the orifice index, $\mathrm{n}=1,2, \ldots$, 16

$t \quad$ Time onset [s]

$u \quad$ Jet velocity $[\mathrm{m} / \mathrm{s}]$

$\overline{U_{j}} \quad$ Mean jet velocity $[\mathrm{m} / \mathrm{s}]$

$\tau \quad$ Periodic time $[\mathrm{s}]$

$\varphi \quad$ Phase angle [deg.]

$\langle\ldots\rangle$ Phase-averaged value of ...

\section{INTRODUCTION}

Previous extensive work on Synthetic Jet Actuator (SJA) technology has proven the great potential of the SJA technology for mitigating or delaying flow separation over critical aerodynamic surfaces, such as airfoils $[1,2]$. Such boundary layer control is achieved through the periodic injection of highmomentum jet(s) into the boundary layer region $[3,4]$. In this technology, typically, a diaphragm is mounted in a sealed cavity which has an orifice leading to the surface where the jet is to be ejected. Imposing periodic oscillations on the diaphragm changes the cavity volume, leading to periodic ingestion and expulsion of the flow through the orifice [5]. The SJA technology is a promising active control technique as it could be finely tuned to generate any required output. 
The synthetic jets are applied to the boundary layer flow commonly through a narrow rectangular slot. Both Amitay et al. [6] and Feero et al. [7] showed successful use of synthetic jets emerging through an orifice that had the shape of a high aspectratio rectangular slot for controlling separation over the span of an airfoil model. The injection of the synthetic jets into the boundary layer region along the span of an airfoil does not have to be from a rectangular slot. For example, Tang et al. [8] achieved separation control using an array of local circular holes distributed over an airfoil span for the ejection of synthetic jets. Be it a spanwise slot or an array of local holes, the physical insight into the flow interactions between the injected jet and the boundary layer region is crucial for understanding the flow control mechanisms. Van Buren et al. [9] investigated a flow field involving synthetic jets, injected through a rectangular slot over a flat plate in crossflow. They reported the formation of two types of flow structures: (a) a main recirculation bubble just downstream of the orifice, and (b) a pair of streamwise vortices that appear far downstream of the jet, which seems to originate from the slot edges. Both structures could be important for flow control and a clearer understanding of the nature of interaction between the synthetic jet and the main flow is still needed.

Feero et al. [10] investigated the effects of SJA operating parameters on the aerodynamic performance of an airfoil having jet expulsion and injection through a large aspect-ratio rectangular slot, spanning the airfoil. These parameters included the actuator excitation frequency, input power, phase-averaged jet velocity, and blowing ratio. The later is quantified by the ratio of time-averaged jet velocity during expulsion half of the cycle to the main flow velocity. They found that, for a blowing ratio less than 1 , effective control can be achieved when the jet excitation frequency matches the shear layer instability. For a blowing ratio more than 1 , the exact matching of frequency to the wake or shear layer instability is not critical and flow control is attained regardless of the frequency value. This explains why most of other studies in the literature target obtaining high jet velocity output from the synthetic jet actuator. The common approach to achieve the highest jet velocity is to drive the synthetic jet actuator at its resonance frequency and to use multiple excitation elements to excite a single large cavity to magnify the output velocity. Understanding the frequency response of such synthetic jet actuators is particularly important to correlate various design parameters (such as the cavity dimensions, and the number and dimensions of orifices) to the output jet velocity.

Hence, the objective of this paper is to investigate the effect of excitation frequency on the mean jet velocity generated by a multi-element SJA design. Focus is placed on synthetic jets emanating from an array of circular orifices, distributed along the span of a flat plate, under quiescent conditions. A thorough review of the literature shows that although the characterization of several operating parameters (including the excitation frequency, excitation amplitude, location of control etc.) on the boundary layer control performance of SJAs has been the focus of significant research activity [10-13], how the design and the operating parameters of array-type actuators affect the synthetized jet velocity along the span of the actuator array has remained unexplored. The work presented herein shows the frequency response resulting from an array-type SJA and how it may be impacted by the cavity design or dimensions.

(a) Synthetic jet actuator

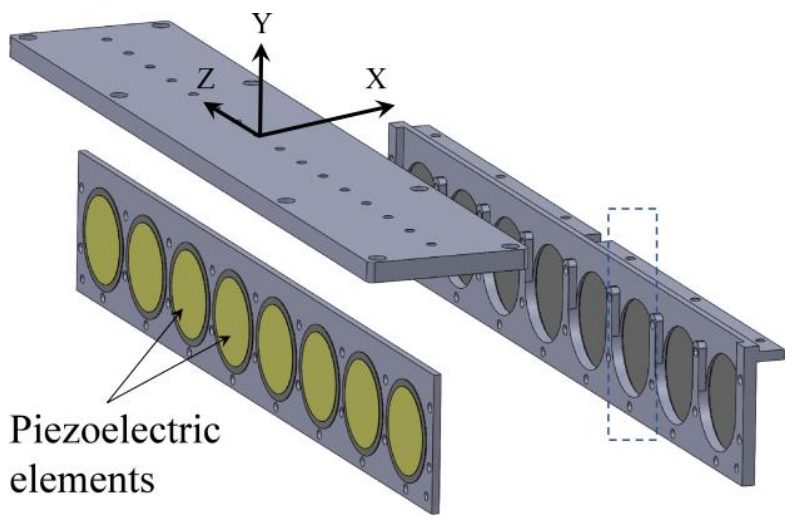

(b) Air volume between each pair of piezoelectric elements

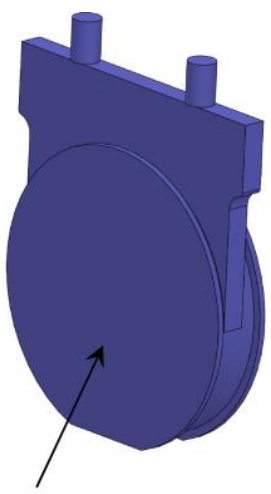

Surface adjacent to

Piezoelectric element

FIGURE 1: (A) EXPANDED VIEW OF THE SYNTHETIC JET ACTUATOR SHOWING THE PIEZOELECTRIC ELEMENTS (8 PAIRS) AND (B) ISOMETRIC VIEW OF THE AIR VOLUME BETWEEN EACH TWO PIEZOELECTRIC ELEMENTS.

\section{EXPERIMENTAL SETUP}

The synthetic jet actuator configuration that is tested in this investigation is comprised of 16 piezoelectric elements that are arranged into 8 pairs mounted on the facing walls of one single cavity, as shown in Fig. 1(a), to excite the cavity simultaneously. The synthetic jets are formed through 16 orifices, distributed over a span of $300 \mathrm{~mm}$ over the face of the cavity that is perpendicular to the sides where the piezoelectric elements are mounted. 
Each orifice has a diameter of $3.42 \mathrm{~mm}$ and the spacing is 19 $\mathrm{mm}$ (center-to-center). The results presented here are for synthetic jets injected to a flat-plate configuration with no crossflow. In Fig. 1(b), the air volume present between any two piezoelectric elements up to the orifice exists is shown. The 16 orifices are situated such that each of the two orifices are centered around the center of each of the piezoelectric elements, as can be seen from Fig. 1(b). It should be noted that the cavity volume consists of 8 repetitions of this shape connected in one unified volume, which is used in acoustic simulations to be presented in this paper. A signal generator is used to power all piezoelectric elements simultaneously by providing discrete sinusoidal excitations at prescribed frequencies. The excitation signal is amplified by a linear power amplifier (Mide Quick- Pack, QPA3202) to provide a constant exctitaion amplitude of $\mathrm{E}=150 \mathrm{~V}_{\mathrm{pp}}$ to all piezoelectric elements. The mean jet velocity is measured by utilizing a hotwire (Dantec miniature probe, 55P11) at the center of each orifice.

\section{RESULTS}

The jet velocity is sampled for 30 seconds at $32 \mathrm{kHz}$. The input excitation signal is also simultaneously recorded to be used as a means for phase-averaging the measured response. Figure 2(a) presents, as a sample data, the phase-averaged velocity measured at the exit of the orifice located in the middle of the array $(\mathrm{n}=8)$, i.e., at $Y / D=0$ and $Z / D=0$ based on the coordinate system introduced in Fig. 1(a). Furthermore, Figure 2(b) shows the corresponding sinusoidal input signal used to excite the piezoelectric elements at a frequency of $f_{e}=1220 \mathrm{~Hz}$ and an amplitude of $\mathrm{E}=150 \mathrm{~V}_{\mathrm{pp}}$. The positive amplitudes in this excitation signal, i.e., $0<\varphi<\pi$, corresponds to the expulsion stroke of the jet, while the negative amplitudes, i.e., $\pi<\varphi<2 \pi$, show the ingestion stroke. The velocity signals detected by the single-wire probe used in this investigation were positive regardless of whether the expulsion or ingestion phase is captured. Hence, the direction of the jet flow cannot be inferred from the hotwire measurements. It is also noticed that the jet velocity over the return stroke seems marginally lower than the expulsion stroke. This is because some of the returning flow is drawn back into the cavity around the orifice edges rather than through the jet core. The error bars in Fig. 2(a) show the standard variation around the phaseaveraged jet velocity at each phase. It is important to point out that the hotwire measurement has a slight lag (or in other words, a phase angle difference) with respect to the input excitation as air viscosity introduces viscous damping to the system. This phase angle difference between the hotwire signal and the excitation input is not significant in the case presented in Fig. 2(a) because it is obtained at a resonant frequency, however cases with other excitation frequencies would exhibit different phase angle differences, as will be shown later in this paper.

The time-averaged mean jet velocity is commonly defined in the literature as the time-averaged value during only the expulsion stroke [12]. It is therefore essential to clearly define the expulsion stroke in the measured velocity signals. In this study, the phase angle corresponding to the maximum jet velocity in the phaseaveraged cycle is used as a reference point, and the phase angle

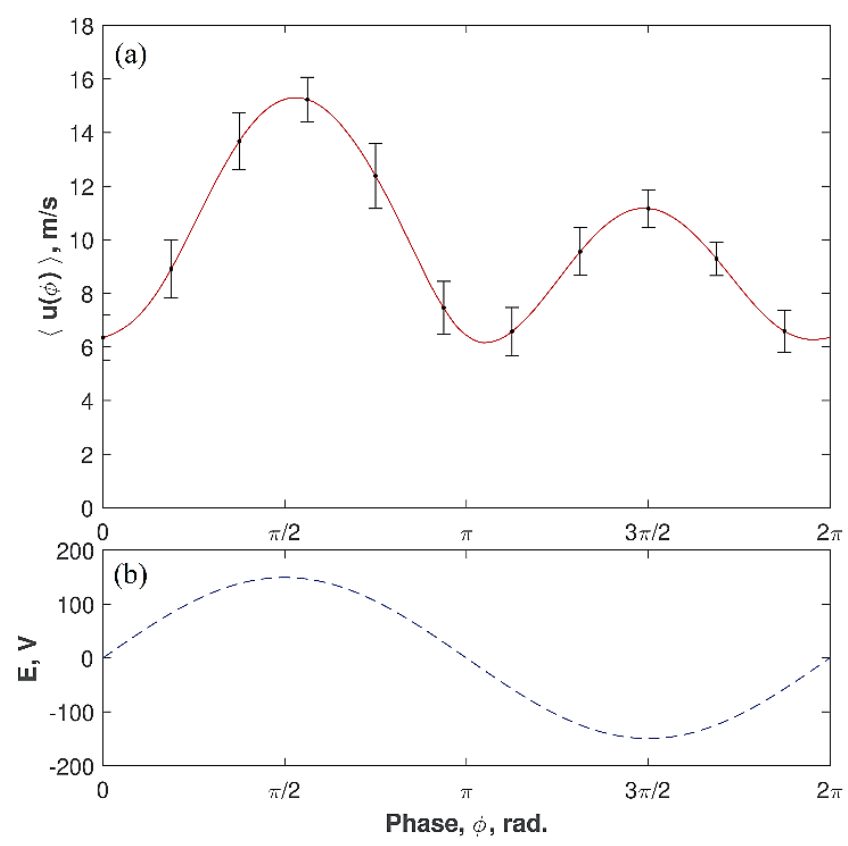

FIGURE 2: (A) PHASE-AVERAGED JET VELOCITY, $\langle u(\Phi)\rangle$, ATTHE EXIT OF THE ORIFICE IN RESPONSE TO (B) THE SINUSOIDAL EXCITATION AT FREQUENCY OF $f_{e}=1220$ HZ AND EXCITATION VOLTAGE OF E $=150 \mathrm{VPP}$. ERROR BARS MARK THE STANDARD DEVIATION OF THE PHASE-AVERAGED MEAN VELOCITY.

range of the expulsion stroke in a cycle is considered to be bounded by a quarter cycle $(\pi / 2)$ before and after the phase of the maximum jet velocity. Based on this, the time-averaged jet velocity during the expulsion stroke will be defined throughout this investigation as:

$$
\overline{U_{j}}=\frac{1}{\tau / 2} \int_{t_{1}}^{t_{2}} u(t) d t
$$

where $\tau$ is the period of one cycle, $t_{1}$ is the onset time of the expulsion stroke and $t_{2}$ is the end time of the expulsion stroke. Here, as the expulsion stroke occurs over half of the cycle, the denominator in equation (1) is $t_{2}-t_{1}=\tau / 2$.

Figure 3 shows the time-averaged jet velocity of the expulsion stroke computed using equation (1) for different excitation frequencies ranging from $f_{e}=500 \mathrm{~Hz}$ to $2000 \mathrm{~Hz}$ at the exit of the orifice that is located in the middle of the array (i.e., at $\mathrm{X} / \mathrm{D}=\mathrm{Z} / \mathrm{D}$ $=\mathrm{Y} / \mathrm{D}=0$ ). This velocity response shows several peaks in the range of $f_{e}=800$ to $1400 \mathrm{~Hz}$. The Helmholtz frequency for the cavity volume can estimated to be $1232 \mathrm{~Hz}$, using the cavity and orifice dimensions [14]. This estimation is very close to the resonant peak observed at $1220 \mathrm{~Hz}$ in the experimental data presented in Fig. 3. Furthermore, it can be seen in Fig. 3 that other than this excitation frequency, three more frequencies exist at which the jet velocity peaks. No known theoretical formulations predict these resonant frequencies in the vicinity of $800-1400 \mathrm{~Hz}$. These observations prompted the idea of measuring the jet velocity at the exit of other orifices to see if those detected resonant frequencies are 


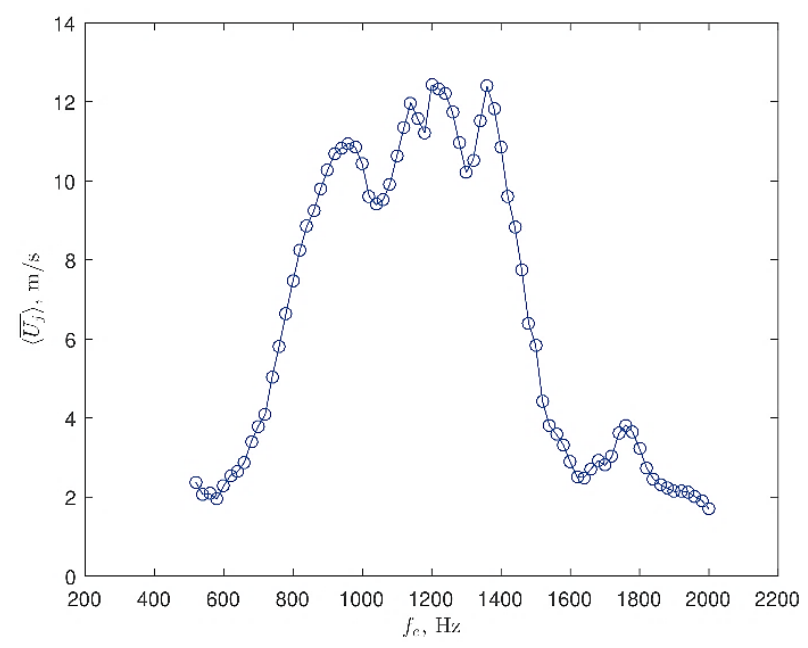

FIGURE 3: TIME-AVERAGED JET VELOCITY, $\left\langle\bar{U}_{j}\right\rangle$, DURING THE PHASE-AVERAGED EXPULSION STROKE AS A FUNCTION OF THE EXCITATION FREQUENCY, $f_{e}$.

independent of the orifice location. When the jet velocity along the array of orifices was captured 1 orifice-diameter above each orifice at the centerline of the jet, a significant variation was observed along the span of the synthetic jet array. Furthermore, the shape of this variation is observed to depend on the excitation frequency, as seen in Fig. 4, where the mean jet velocity over each orifice is depicted for two excitation frequencies (the resonance frequency of $1200 \mathrm{~Hz}$ and off-resonance frequency of $800 \mathrm{~Hz}$ ). It can be seen from this figure that when the SJA array is excited near the Helmholtz frequency of $1200 \mathrm{~Hz}$, the mean jet velocity increases over two sections: namely, over the orifices indexed as $n=3$ to 6 and $n=10$ to 14 . On the contrary, when the excitation is supplied at a frequency of $800 \mathrm{~Hz}$, which is away from any resonant peaks, the orifices indexed as $n=3$ to 6 have a marginal increase while the orifices indexed as $n=10$ to 14 undergo a significant reduction in velocity.

As the pattern of variation in the mean jet velocity along the array of orifices depends on the value of the excitation frequency (as discussed above), acoustic simulations are performed to investigate the acoustic mode shapes inside the cavity. COMSOL Multi-physics software is used in the present study to perform an eigen-frequency study for the internal cavity volume. Solving the wave equation for the eigen-frequencies and the eigen-modes gives the resonance frequencies and the corresponding acoustic mode shapes, respectively [15]. Care is given to the meshing technique where unstructured tetrahedron elements with various sizes are used to finely capture all details of the internal cavity geometry. The acoustic simulations gave several eigen-frequencies that closely match the frequency peaks detected experimentally from the jet velocity measurements, as shown in Tab. 1. Figure 5 shows the acoustic pressure distribution within the cavity volume for the resonance frequency at $1252 \mathrm{~Hz}$. These acoustic pressure levels, generated by simulations, are arbitrary, and therefore, were normalized by the maximum pressure level detected in the cavity for any given case. From Fig. 5, it can be

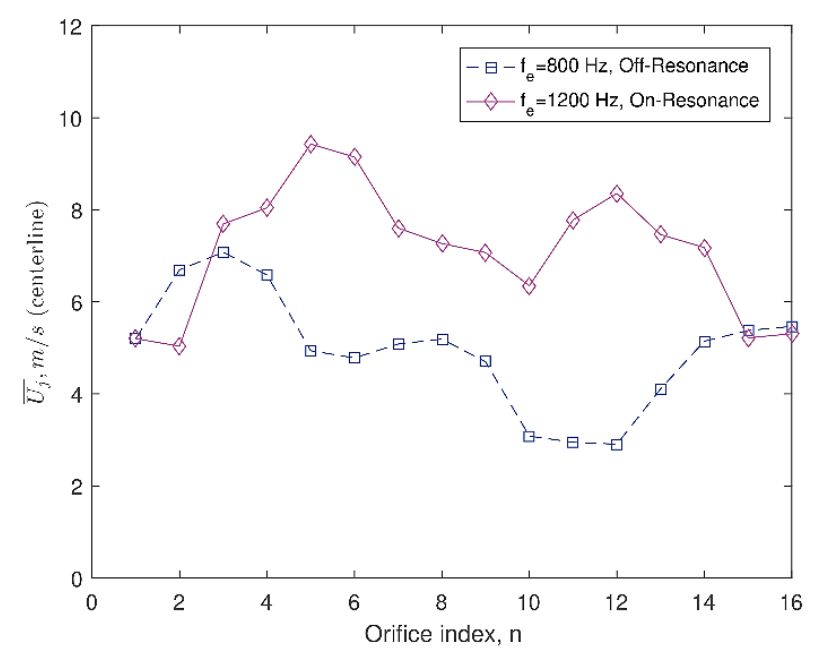

FIGURE 4: THE VARIATION OF THE MEAN JET VELOCITY ALONG THE ARRAY OF ORIFICES AT Y/D $=1$ (I.E., 1 ORIFICE DIAMETER ABOVE EACH ORIFICE) FOR TWO EXCITATION FREQUENCIES $\left(f_{e}=800 \mathrm{HZ}\right.$ AND $\left.1200 \mathrm{HZ}\right)$.

TABLE 1: COMPARISON OF THE RESONANT FREQUENCIES, in Hz, OBTAINED FROM ACOUSTIC SIMULATIONS WITH THOSE EXTRACTED FROM EXPERIMENTAL MEASUREMENTS.

\begin{tabular}{ccccc}
\hline Mode index & 1 & 2 & 3 & 4 \\
\hline Simulations & 956 & 1126 & 1252 & 1331 \\
Experiments & 960 & 1140 & 1220 & 1360 \\
\hline
\end{tabular}

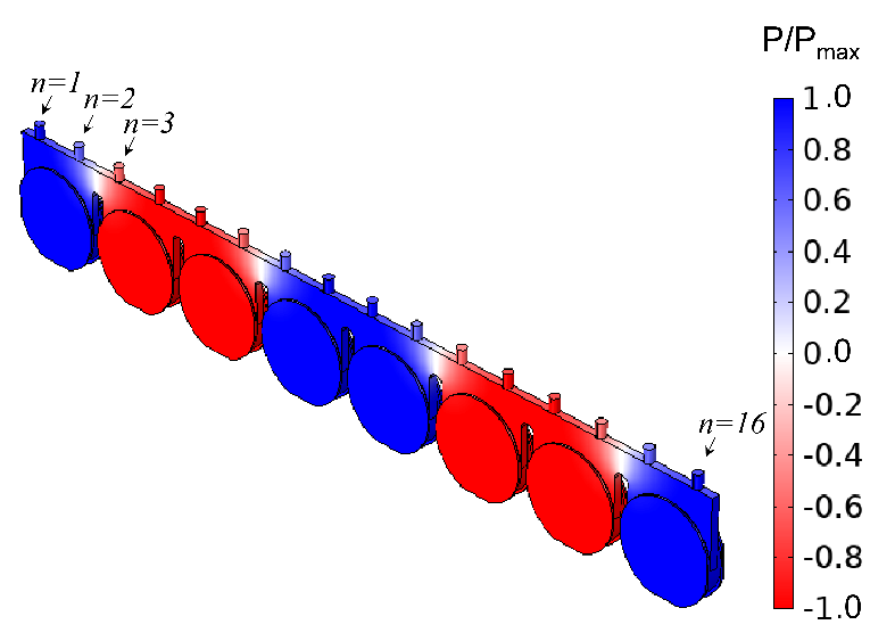

FIGURE 5: ACOUSTIC MODE SIMULATIONS SHOWING ACOUSTIC PRESSURE VARIATION IN THE CAVITY AT THE RESONANCE FREQUENCY, $f_{S}=1252 \mathrm{HZ}$.

seen that, for the resonance frequency at of $1252 \mathrm{~Hz}$, two portions of the cavity volume exhibit higher acoustic pressure distributions (shown with red contours) compared to cavity portions at the two ends and in the middle (shown in blue). These results agree well with the experimentally observed variation of mean jet velocity at the exit of each orifice for the excitation frequency of 


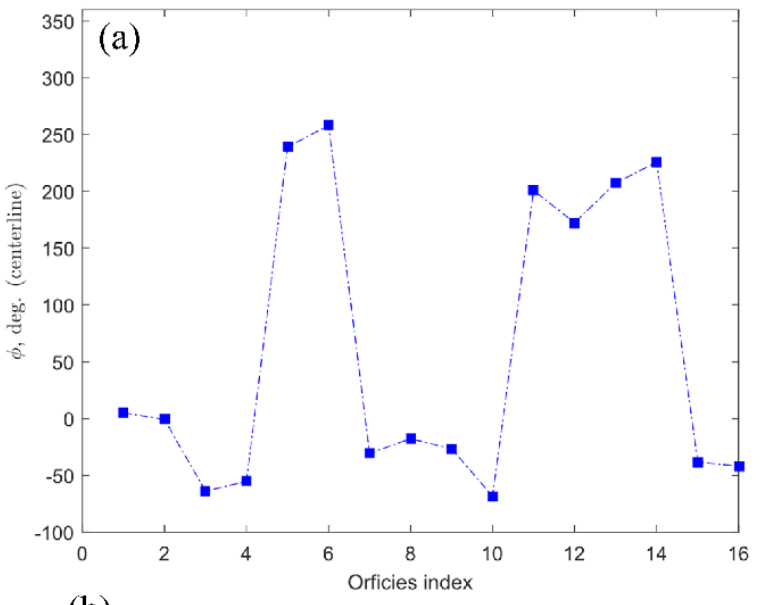

(b)

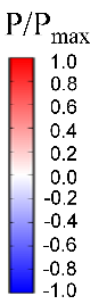

FIGURE 6: (A) THE VARIATION OF THE PHASE ANGLE DIFFERENCE BETWEEN THE PHASE-AVERAGED PEAK JET VELOCITY AND THE INPUT EXCITATION ALONG ALL 16 ORIFICES FOR EXCITATION AT THE RESONANT FREQUENCY, OF $f e=1220 \mathrm{HZ}$. THE JET VELOCITIES ARE MEASURED 1 ORIFICE DIAMETER ABOVE EACH ORIFICE, AT THE CENTER OF EACH JET. (B) THE VARIATION OF THE NORMALIZED ACOUSTIC PRESSURE WITHIN THE CAVITY VOLUME FOR THE CORRESPONDING ACOUSTIC MOD, AT RESONANT FREQUENCY, $f s=1252 \mathrm{HZ}$.

$f e=1200 \mathrm{~Hz}$, shown earlier in Fig. 4 .

To obtain further insight into the correlation between the excited acoustic mode shape of the cavity and the jet velocities emanating from orifices, the phase angle difference of the phaseaveraged jet velocity between the orifices of the SJA array is determined. As explained earlier, the phase angle difference can be extracted from the phase-averaged jet velocity measurements by comparing the phase of the highest phase-averaged jet velocity of the expulsion stroke to the phase of the maximum voltage of the sinusoidal excitation, as explained earlier in Fig. 2. Since the input excitation was recorded simultaneously with the measured jet velocity, phase-averaged correlation could easily be extracted and compared for different excitation frequencies, for which the excitation amplitude was kept constant.

The excited mode shape corresponding to the case shown earlier in Fig. 2, at $f_{e}=1220 \mathrm{~Hz}$, exhibits an acoustic anti-node around the middle of the span, i.e. around $n=8$ to 9 , as evident from Fig. 6(b). The phase angle difference between the phaseaveraged jet velocity and the input excitation in this case is around 0 degrees, as seen in Fig. 6(a), which is extracted from the localized jet measurements. Figure 6(b) also shows that the acoustic pressure level is higher for this case in two cavity regions

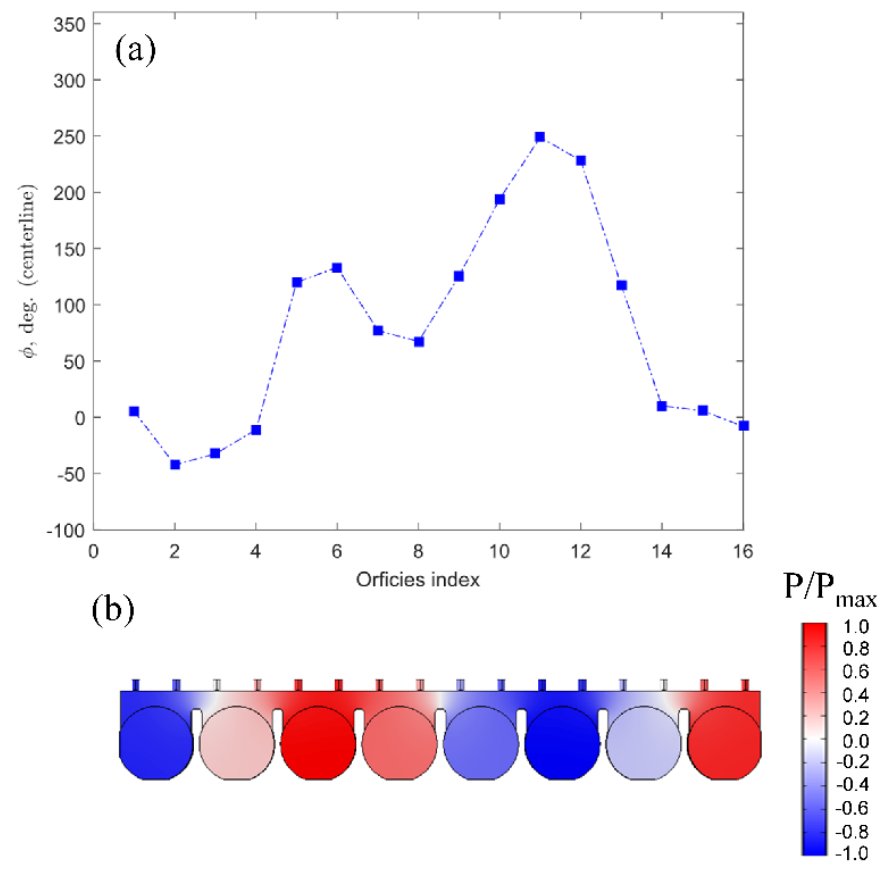

FIGURE 7: (A) THE VARIATION OF THE PHASE ANGLE DIFFERENCE BETWEEN THE PHASE-AVERAGED PEAK JET VELOCITY AND THE INPUT EXCITATION ALONG ALL 16 ORIFICES FOR EXCITATION AT THE FREQUENCY OF $f e=800 \mathrm{HZ}$, WHICH IS AWAY FROM ANY RESONANT PEAK. (B) THE VARIATION OF THE NORMALIZED ACOUSTIC PRESSURE WITHIN THE CAVITY VOLUME FOR THE CLOSEST ACOUSTIC MODE

(indicated with red contours), which contain the orifices $n=3$ to 6 and $n=10$ to 14 . For these same orifices, the phase angle difference between the jet velocity and the input excitation is non-zero, as can be seen from Fig. 6(a). However, both regions are in-phase with each other, which further explains why the jet velocities measured for the orifices in these two regions are relatively higher than the velocities in the middle region and far sides, which were observed earlier in Fig. 4.

At the off-resonance excitation frequency of $f_{e}=800 \mathrm{~Hz}$, the acoustic pressure distribution locks into the closest acoustic mode shape shown in Fig. 7(b). For this mode shape, there seem to be two alternating out-of-phase acoustic pressure regions, shown by the blue and red regions in Fig. 7(b). The phase angle variation in the phase-averaged jet velocity shown in Fig. 7(a) depicts that the two regions containing the orifices $n=3-6$ and $n=10-14$ are almost 90 degrees out of phase. It should be emphasized here that the value of the phase angle itself is irrelevant, as the phase-averaged peak jet velocities of all orifices are referenced to the same phase of the input excitation. The fact that the two regions are out of phase of each other explains why there would be a difference in the shape of the jet velocity measured above the orifices along the span. These results point out the challenge of achieving a uniform synthetic jet injection along the plate span for optimum flow control. 


\section{CONCLUSION}

The effect of excitation frequency on synthetic jet velocity was experimentally and numerically investigated for an array of circular orifices, distributed over a single cavity excited by 16 piezoelectric elements. Several excitation frequencies are observed to yield peaks in the time-averaged jet velocity of the expulsion stroke. For both on- and off-resonance excitations, the time-averaged jet velocity of the expulsion stroke varies between orifices. Acoustic simulations of the cavity volume show that each resonant frequency corresponds to an acoustic mode shape of the cavity volume. The different mode shapes result in a distinct acoustic pressure distribution within the cavity, which, in turn, leads to a corresponding variation in the jet velocities from one orifice to another within the array. Moreover, the phase variations of the jet velocity along the orifices are linked to the acoustic mode shapes of the cavity. Jet velocity and phase angle variations observed along orifices point out possible challenges in developing uniform control schemes across airfoils using an array of circular orifices from a single chamber.

\section{ACKNOWLEDGMENT}

This work has been funded by the internal Dean Strategic Fund from the Faculty of Applied Science and Engineering of the University of Toronto (DSF 19-30). The authors greatly appreciate this fund that fosters collaborations between different departments within the University of Toronto.

\section{REFERENCES}

[1] Glezer, A., Amitay, M., and Honohan, A. M., 2005. "Aspects of low-and high-frequency actuation for aerodynamic flow control". AIAA Journal, 43(7), pp. 1501-1511.

[2] Seifert, A., Darabi, A., and Wyganski, I., 1996. "Delay of airfoil stall by periodic excitation". Journal of aircraft, 33(4), pp. 691-698.

[3] Cattafesta III, L. N., and Sheplak, M., 2011. "Actuators for active flow control". Annual Review of Fluid Mechanics, 43, pp. 247-272.

[4] Goodfellow, S. D., Yarusevych, S., and Sullivan, P. E., 2013. "Momentum coefficient as a parameter for aerodynamic flow control with synthetic jets". AIAA Journal, 51(3), pp. 623-631.

[5] Smith, B. L., and Glezer, A., 1998. "The formation and evolution of synthetic jets". Physics of fluids, 10(9), pp. 2281- 2297.

[6] Amitay, M., Smith, D. R., Kibens, V., Parekh, D. E., and Glezer, A., 2001. "Aerodynamic flow control over an unconventional airfoil using synthetic jet actuators". AIAA Journal, 39(3), pp. 361-370.

[7] Feero, M. A., Goodfellow, S. D., Lavoie, P., and Sullivan,
P. E., 2015. "Flow reattachment using synthetic jet actuation on a low-Reynolds-number airfoil". AIAA Journal, 53(7), pp. 2005-2014.

[8] Tang, H., Salunkhe, P., Zheng, Y., Du, J., and Wu, Y., 2014. "On the use of synthetic jet actuator arrays for active flow separation control". Experimental thermal and fluid science, 57, pp. 1-10.

[9] Van Buren, T., Beyar, M., Leong, C. M., and Amitay, M., 2016. "Three-dimensional interaction of a finite-span synthetic jet in a crossflow". Physics of Fluids, 28(3), p. 037105.

[10] Feero, M. A., Lavoie, P., and Sullivan, P. E., 2017. "Influence of synthetic jet location on active control of an airfoil at low-Reynolds number". Experiments in Fluids, 58(8), p. 99.

[11] Chaudhari, M., Verma, G., Puranik, B., \& Agrawal, A. (2009). Frequency response of a synthetic jet cavity. Experimental thermal and fluid science, 33(3), 439-448.

[12] Holman, R., Utturkar, Y., Mittal, R., Smith, B. L., and Cattafesta, L., 2005. "Formation criterion for synthetic jets". AIAA Journal, 43(10), pp. 2110-2116.

[13] Glezer, A., \& Amitay, M. (2002). Synthetic jets. Annual review of fluid mechanics, 34(1), 503-529.

[14] Gallas, Q., Holman, R., Nishida, T., Carroll, B., Sheplak, M., and Cattafesta, L., 2003. "Lumped element modeling of piezoelectric-driven synthetic jet actuators". AIAA Journal, 41(2), pp. 240-247.

[15] Kinsler, L. E., Frey, A. R., Coppens, A. B., \& Sanders, J. V. (1999). Fundamentals of Acoustics. John Wiley \& sons. 